STRUCTURAL BIOLOGY COMMUNICATIONS

ISSN 2053-230X

\section{Crystal structure of a short-chain dehydrogenase/ reductase from Burkholderia phymatum in complex with NAD}

\author{
Jawaher Alenazi, ${ }^{a}$ Stephen Mayclin, ${ }^{\mathrm{b}, \mathrm{c}}$ Sandhya Subramanian, ${ }^{\mathrm{c}, \mathrm{d}}$ Peter J. Myler ${ }^{\mathrm{c}, \mathrm{d}}$ \\ and Oluwatoyin A. Asojo ${ }^{a_{*}}$
}

Received 26 October 2021

Accepted 6 January 2022

Edited by N. Sträter, University of Leipzig, Germany

Keywords: SSGCID; oxidoreductases; Burkholderia phymatum; short-chain dehydrogenase/reductase family; NAD; structural genomics; Seattle Structural Genomics Center for Infectious Disease.

PDB reference: short-chain dehydrogenase/ reductase from Burkholderia phymatum in complex with NAD, 5 ig2

Supporting information: this article has supporting information at journals.iucr.org/f

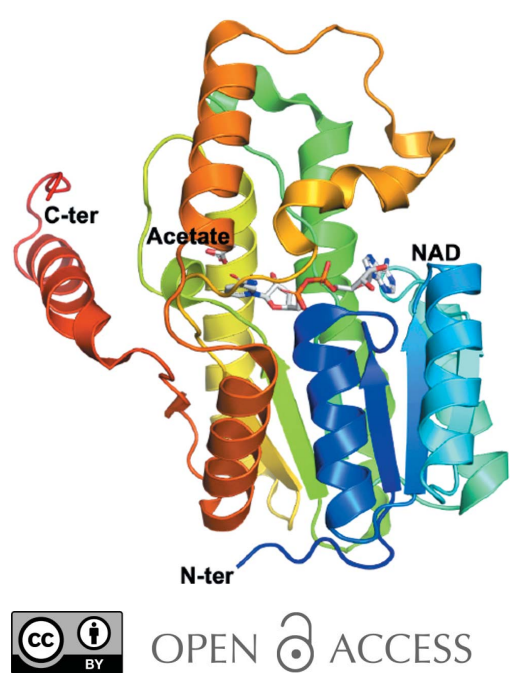

aDepartment of Chemistry and Biochemistry, Hampton University, 200 William R. Harvey Way, Hampton, VA 23668,

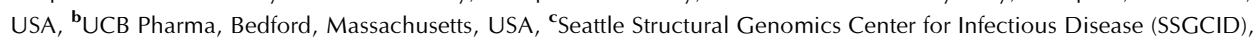
Seattle, Washington, USA, and ' Center for Global Infectious Disease Research, Seattle Children's Research Institute, 307 Westlake Avenue North Suite 500, Seattle, Washington, USA. *Correspondence e-mail:

oluwatoyin.asojo@hamptonu.edu

Burkholderia phymatum is an important symbiotic nitrogen-fixing betaproteobacterium. B. phymatum is beneficial, unlike other Burkholderia species, which cause disease or are potential bioagents. Structural genomics studies at the SSGCID include characterization of the structures of short-chain dehydrogenases/reductases (SDRs) from multiple Burkholderia species. The crystal structure of a short-chain dehydrogenase from B. phymatum (BpSDR) was determined in space group $C 222_{1}$ at a resolution of $1.80 \AA$. BpSDR shares less than $38 \%$ sequence identity with any known structure. The monomer is a prototypical SDR with a well conserved cofactor-binding domain despite its low sequence identity. The substrate-binding cavity is unique and offers insights into possible functions and likely inhibitors of the enzymatic functions of $B p S D R$.

\section{Introduction}

Burkholderia are nonfermenting motile Gram-negative bacteria that are among the largest groups of species of Betaproteobacteria and include infective and symbiotic species (Yabuuchi et al., 1992; Sawana et al., 2014). Burkholderia can cause serious infections in humans; for example, B. pseudomallei causes melioidosis, a deadly emerging opportunistic infection (Hall et al., 2019; Poe et al., 1971; Veluthat et al., 2021), and B. glumae and B. gladioli cause infections in plants (Zhou-qi et al., 2016). B. phymatum was identified in root-nodule isolates from tropical legumes and is capable of symbiotic nitrogen fixation with the legume Machaerium lunatum and Mimosa pudica (Vandamme et al., 2002). Due to the importance of Burkholderia, there is a need to clarify the structures of enzymes that play important roles in the life cycles of these bacteria. The SSGCID has characterized the structures of proteins that may play important roles in these bacteria. The short-chain dehydrogenase/ reductases (SDRs) are $\mathrm{NAD}(\mathrm{P})(\mathrm{H})$-dependent oxidoreductases that may be involved in the metabolism of diverse cofactors, hormones and xenobiotic or other compounds. The protein structure reported here is a putative SDR which shares less than $38 \%$ sequence identity with any published structure. This structure is part of structural genomics efforts at the Seattle Structural Genomics Center for Infectious Disease (Raymond et al., 2011; Myler et al., 2009; Baugh et al., 2013). This manuscript was completed in a partnership between Hampton University and the SSGCID (Asojo, Dranow et al., 2018; Asojo, Subramanian et al., 2018). molecules, including lipids, amino acids, carbohydrates, 
Table 1

Macromolecule-production information.

\begin{tabular}{ll}
\hline Source organism & $\begin{array}{c}\text { Burkholderia phymatum (strain DSM } \\
\text { 17167/STM815) } \\
\text { Genomic DNA }\end{array}$ \\
DNA source & $5^{\prime}$-CTGCGAAAGCGGAT-3' \\
Forward primer & $5^{\prime}$-TGCCATACTCTAGCYYGC-3' \\
Reverse primer & pBG1861 \\
Expression vector & E. coli BL21 (DE3) \\
Expression host & MAHHHHHHFEFDGKVAVITGAGSGFGRAF \\
Complete amino-acid sequence & AEKGASLGMKLVLADVDEGALARTVDTL \\
of the construct produced & RAAGAEVIGVRTDVSGAQVQAADAAL \\
& EAFGKVHLLFNAGVGAGGFLESSAND \\
& WAWVFGVNVMGVAHGVRVAPIMLGQNE \\
& AAHIVNTASVAGLSPPSMGIYNASKHA \\
& VVSLTETLYHDLRNAGGEVGSLLPAF \\
& VPTGIADAERVRPEALRNEAQPTRSQLA \\
& ADRQLQRAVRSGKLGATDVATLTFEAIA \\
& ERRFYILTHPALATVRLRHEDIELQRN \\
& PTDPLSLKPEVKEAR \\
\hline
\end{tabular}

Table 2

Crystallization.

\begin{tabular}{ll}
\hline Method & Vapor diffusion, sitting drop \\
Temperature (K) & 287 \\
Protein concentration $\left(\mathrm{mg} \mathrm{ml}^{-1}\right)$ & 45 \\
Buffer composition of protein & $300 \mathrm{~m} M \mathrm{NaCl}, 20 \mathrm{~m} M$ HEPES, \\
$\quad$ solution & $5 \%$ glycerol, $1 \mathrm{~m} M$ TCEP pH 7.0 \\
Composition of reservoir & $\mathrm{MCSG} 1 \mathrm{C} 4: 170 \mathrm{~m} M$ ammonium acetate, \\
solution & $85 \mathrm{~m} M$ sodium acetate-HCl $\mathrm{pH} 4.6$, \\
& $25.5 \%(w / v)$ PEG 4000, 15\% $(v / v)$ \\
& glycerol, $4 \mathrm{~m} M$ NAD \\
Volume and ratio of drop & $0.4 \mu \mathrm{l}: 0.4 \mu \mathrm{l}$
\end{tabular}

\section{Materials and methods}

\subsection{Macromolecule production}

SDR from $B$. phymatum (BpSDR) was produced at the SSGCID (Myler et al., 2009; Stacy et al., 2011) following standard protocols described previously (Bryan et al., 2011; Choi et al., 2011; Serbzhinskiy et al., 2015). Dr Mary Lidstrom of the University of Washington provided genomic DNA from B. phymatum STM815 (Moulin et al., 2014). The sequence (UniProt ID B2JGP2, GenBank ID ACC70227.1) encoding amino acids 1-289 was PCR-amplified from genomic DNA using the primers shown in Table 1. The resultant amplicon was cloned by ligation-independent cloning into pET-14b expression vector pBG1861 provided by Dr Wesley Van Voorhis of the University of Washington. The vector encodes a noncleavable hexahistidine fusion tag (MAHHHHHHMORF). The plasmid DNA was transformed into chemically competent Escherichia coli BL21(DE3) cells (Table 1).

Recombinant $B p$ SDR was purified by the standard two-step protocol consisting of immobilized metal-affinity chromatography (IMAC) followed by size-exclusion chromatography (SEC) at SSGCID. All chromatography runs were performed on an ÄKTApurifier 10 (GE) using automated IMAC and SEC programs as described previously (Bryan et al., 2011). Specifically, SEC was performed on a HiLoad 26/600 Superdex 75 column (GE Healthcare) using a mobile phase consisting of $300 \mathrm{~m} M \mathrm{NaCl}, 20 \mathrm{~m} M$ HEPES, 5\% glycerol, $1 \mathrm{~m} M$ TCEP $\mathrm{pH}$ 7.0. BpSDR eluted as a single peak with a projected molecular weight of $22 \mathrm{kDa}$, indicating that the protein could either be a
Table 3

Data collection and processing.

Values in parentheses are for the outer shell.

\begin{tabular}{ll}
\hline Diffraction source & APS beamline 21-ID-F \\
Wavelength $(\AA)$ & 0.97872 \\
Temperature $(\mathrm{K})$ & 100 \\
Detector & RayoniX MX-225 CCD \\
Space group & $C 222_{1}$ \\
$a, b, c(\AA)$ & $83.52,187.79,108.26$ \\
$\alpha, \beta, \gamma\left({ }^{\circ}\right)$ & $90,90,90$ \\
Resolution range $(\AA)$ & $46.895-1.800(1.850-1.800)$ \\
Total No. of reflections & 490706 \\
Completeness $(\%)$ & $99.900(99.900)$ \\
Multiplicity & $6.220(6.23)$ \\
$\langle I / \sigma(I)\rangle$ & $20.630(3.69)$ \\
$R_{\text {r.i.m. }}$ & $0.071(0.591)$ \\
Overall $B$ factor from Wilson plot $\left(\AA^{2}\right)$ & 18.400 \\
\hline
\end{tabular}

monomer or a dimer in solution. Protein purity was assessed using a reduced SDS-PAGE gel. The peak fractions were concentrated to $44.8 \mathrm{mg} \mathrm{ml}^{-1}$ using an Amicon Ultra-15 30K molecular-weight cutoff concentrator (Millipore, Billerica, Massachusetts, USA). Aliquots of $200 \mu \mathrm{l}$ were flash-frozen in liquid nitrogen and stored at $-80^{\circ} \mathrm{C}$ until use for crystallization. The pure protein is available at https://apps.sbri.org/ SSGCIDTargetStatus/Target/BuphA.00010.n, as are the expressing clones.

\subsection{Crystallization}

$B p S D R$ was crystallized using the sitting-drop vapordiffusion method. Prior to crystallization, NAD was added to the protein stock to a final concentration of $4 \mathrm{~m} M$. Crystallization drops consisting of $0.4 \mathrm{ml} \mathrm{BpSDR}\left(45 \mathrm{mg} \mathrm{ml}^{-1}\right)$ and $0.4 \mathrm{ml}$ precipitant were equilibrated against $80 \mathrm{ml}$ precipitant in the reservoir at $14^{\circ} \mathrm{C}$. Crystals were obtained using condition C4 from the MCSG1 sparse-matrix screen [170 $\mathrm{mM}$ ammonium acetate, $85 \mathrm{~m} M$ sodium acetate- $\mathrm{HCl} \mathrm{pH} 4.5$, $25 \%(w / v)$ PEG $4000,15 \%(v / v)$ glycerol] as the precipitant (Table 2). Upon harvesting, crystals were cryocooled by plunging them into liquid nitrogen without additional cryoprotection.

\subsection{Data collection and processing}

X-ray diffraction data were collected on LS-CAT beamline 21-ID-F at the Advanced Photon Source (APS). Diffraction data (Table 3) were integrated using $X D S$ and reduced using XSCALE (Kabsch, 2010). Data quality was assessed using POINTLESS (Evans, 2006). Raw diffraction data images are available at https://proteindiffraction.org/project/5ig2/.

\subsection{Structure solution and refinement}

The $B p S D R$ structure was solved by molecular replacement (MR) using MOLREP (Lebedev et al., 2008; Vagin \& Teplyakov, 2010). The MR search model was the structure of a Rv0851c ortholog short-chain dehydrogenase from Mycobacterium paratuberculosis (PDB entry 3tjr; Baugh et al., 2015), the structure with the closest amino-acid sequence identity to BpSDR (38\% at over $89 \%$ coverage). Model building and structure refinement were performed using 
Table 4

Structure refinement.

Values in parentheses are for the outer shell.

\begin{tabular}{ll}
\hline Resolution range $(\AA)$ & $46.8950-1.8000(1.8530-1.8000)$ \\
Completeness (\%) & 99.9 \\
$\sigma$ Cutoff & $F>1.340 \sigma(F)$ \\
No. of reflections, working set & $77190(6318)$ \\
No. of reflections, test set & $1674(128)$ \\
Final $R_{\text {cryst }}$ & $0.141(0.2382)$ \\
Final $R_{\text {free }}$ & $0.177(0.2715)$ \\
No. of non-H atoms & \\
$\quad$ Protein & 6141 \\
$\quad$ Ion & 12 \\
$\quad$ Ligand & 132 \\
$\quad$ Solvent & 845 \\
$\quad$ Total & 7130 \\
R.m.s. deviations & \\
$\quad$ Bonds $(\AA)$ & 0.006 \\
$\quad$ Angles $\left({ }^{\circ}\right)$ & 0.777 \\
Average $B$ factors $\left(\AA^{2}\right)$ & \\
$\quad$ Protein & 21.6 \\
Ion & 47.4 \\
$\quad$ Ligand & 15.8 \\
$\quad$ Water & 33.7 \\
Ramachandran plot & \\
$\quad$ Most favored $(\%)$ & 99 \\
Allowed $(\%)$ & 1 \\
\hline
\end{tabular}

iterative cycles of Coot (Emsley et al., 2010) and Phenix (Liebschner et al., 2019). The quality of the model was assessed using MolProbity (Chen et al., 2010). Figures were prepared using PyMOL (DeLano, 2002). The resulting structure-refinement data are provided in Table 4.

\section{Results and discussion}

The crystal structure of $B p S D R$ was solved in space group $C 222_{1}$ with three monomers in the asymmetric unit (Fig. 1). The three monomers of BpSDR are very similar (with an r.m.s.d. of $0.119 \AA$ for the alignment of all $\mathrm{C}^{\alpha}$ atoms) and have the prototypical SDR topology (Fig. 1). Three dimers consistent with prototypical SDR dimers are easily generated by symmetry analysis (Fig. 1b). The buried surface interface for each prototypical SDR dimer is large, including over 50 amino acids and covering $\sim 2640 \AA^{2}$ per monomer. BpSDR is a ternary complex, with each monomer having an acetate molecule in the substrate-binding domain and a NAD molecule in the cofactor-binding domain (Fig. 2a). The cofactorbinding domain is the largest central cavity in the structure, and it is connected to the substrate-binding domain (Fig. 2b).

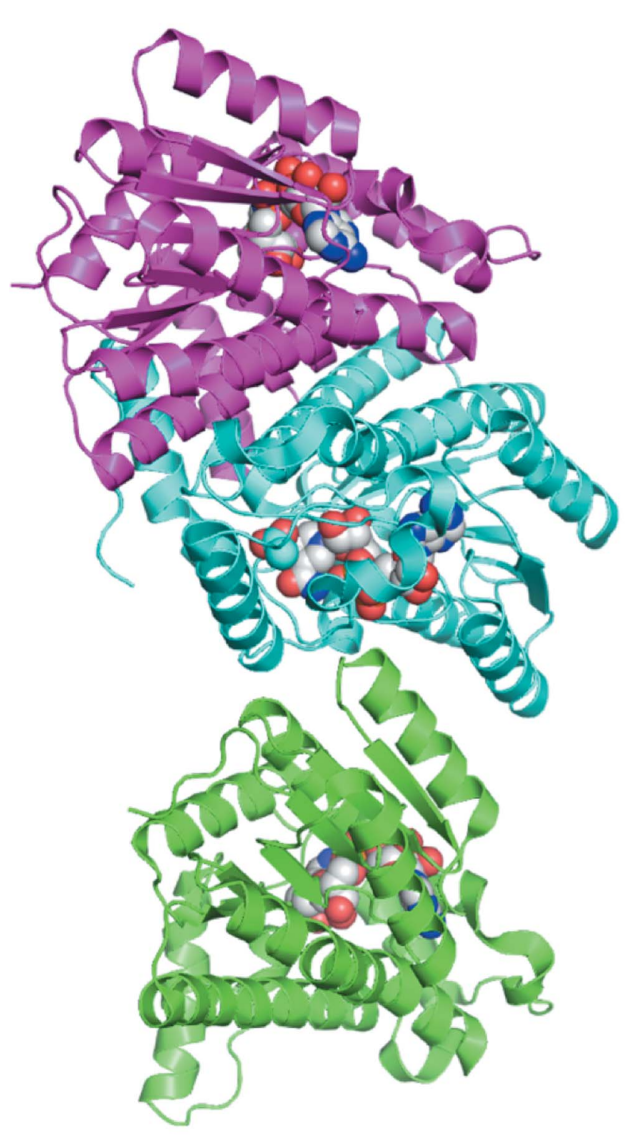

(a)

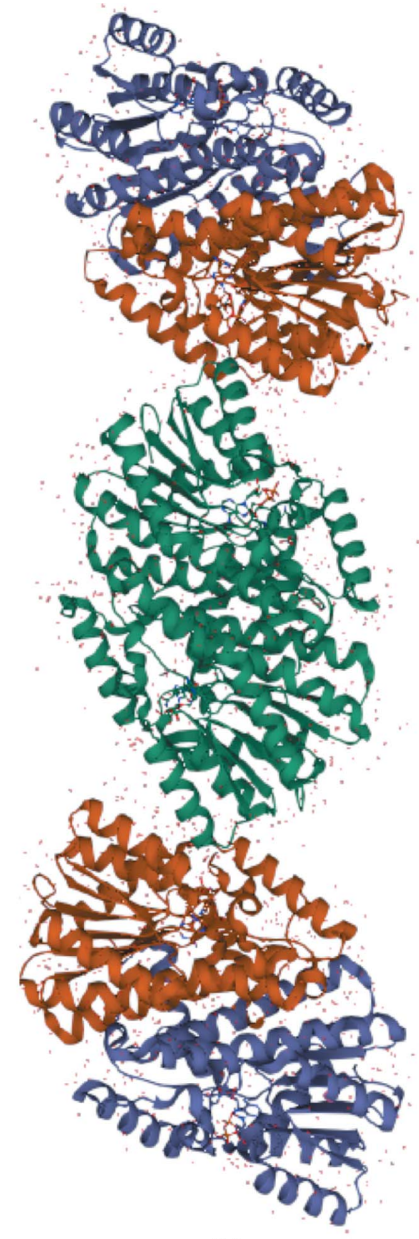

(b)

Figure 1

Crystal structure of BpSDR. (a) A ribbon diagram of BpSDR shows three monomers, colored magenta, cyan and aquamarine (NAD is shown as spheres), in the asymmetric unit. $(b)$ Three prototypical SDR dimers can be generated from the $B p S D R$ structure (the red dots represent water molecules and ligands are shown as sticks). 
The 278 amino acid residues in each monomer have a secondary structure consisting of $12.2 \% \quad \beta$-strand, $47.8 \%$ $\alpha$-helix and $6.5 \% 33_{10}$-helix and have the five $\beta$ - $\alpha$ - $\beta$ motifs of a prototypical SDR (Fig. 2c).

The most similar structures to BpSDR were identified using PDBeFold analysis (http://www.ebi.ac.uk/msd-srv/ssm) at the default threshold cutoff of $70 \%$. PDBeFold analysis revealed that the MR search model (PDB entry 3tjr; Baugh et al., 2015) has the highest structural similarity to $B p S D R$. To compare the substrate-binding cavities and determine whether the small molecules that bind to similar SDRs can bind to BpSDR, the closest structures with bound ligands in their substrate-binding domain were selected from the results of PDBeFold analysis.
The inhibitor-bound structures identified from this analysis include PDB entry $1 \mathrm{fmc}$ ( $7 \alpha$-hydroxysteroid dehydrogenase in complex with NADH and 7-oxoglycochenodeoxycholic acid; Tanaka et al., 1996), PDB entry 4yai (Ligl from Sphingobium sp. strain SYK-6 in complex with NADH and GGE; Pereira et al., 2016), PDB entry 2jap (clavulanic acid dehydrogenase; MacKenzie et al., 2007), PDB entry 6g4l (17 $\beta$-hydroxysteroid dehydrogenase type 14 mutant $\mathrm{Y} 253 \mathrm{~A}$ in complex with a nonsteroidal inhibitor; Badran et al., 2019) and PDB entry 3tjr (crystal structure of the Rv0851c ortholog short-chain dehydrogenase from M. paratuberculosis; Baugh et al., 2015). The superposed structures reveal conserved cofactor-binding domains and varied substrate-binding cavities (Figs. 3 and 4).

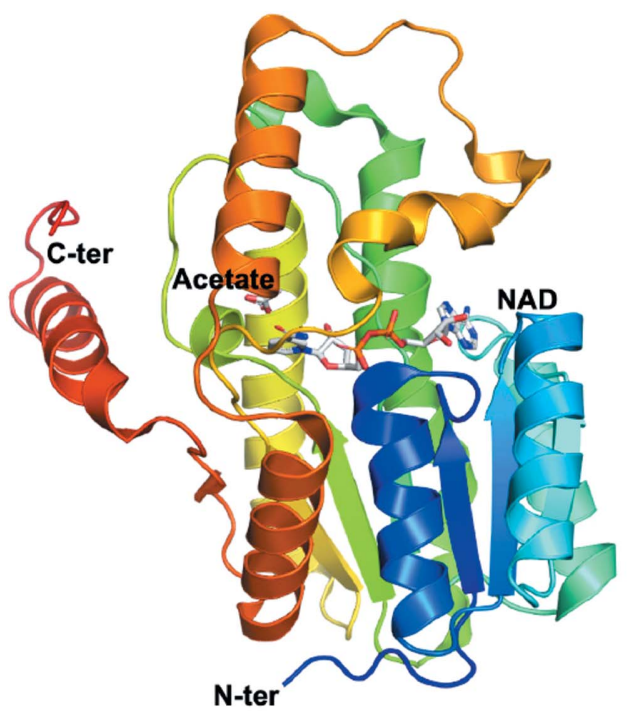

(a)

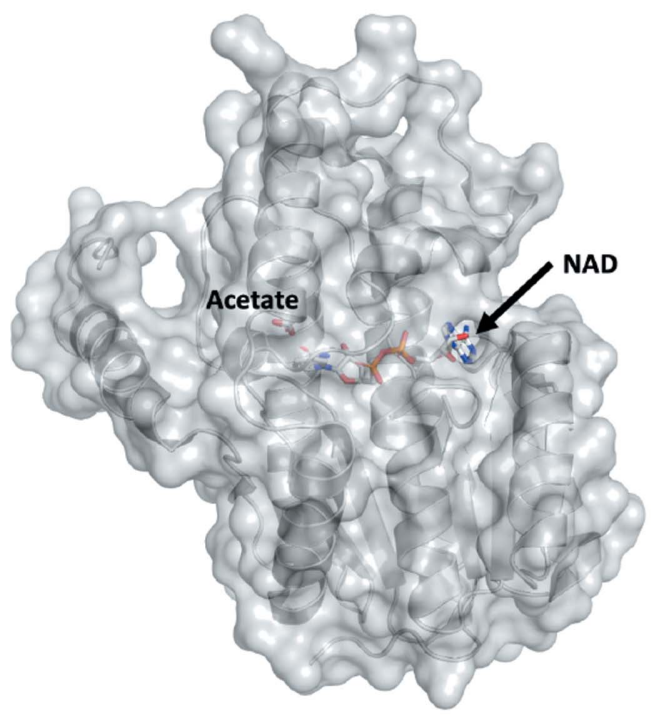

(b)

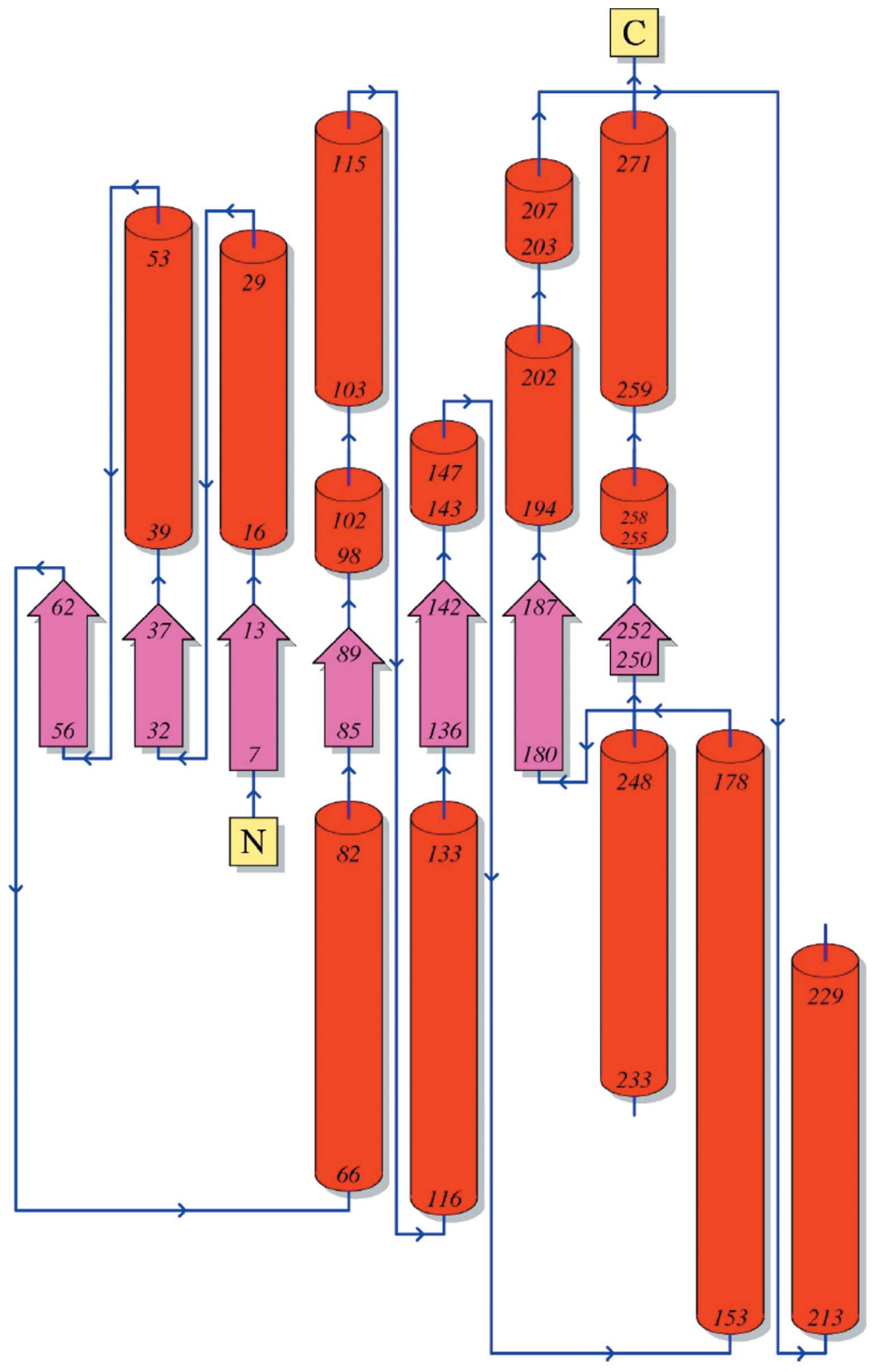

(c)

Figure 2

(a) Monomer structure of $B p$ SDR (PDB entry 5ig2) in a ternary complex with NAD and acetate. NAD binds in the cofactor-binding cavity, while acetate from the crystallization solution is in the substrate-binding site. The monomer is rainbow-colored from the $\mathrm{N}$-terminus (blue) to the $\mathrm{C}$-terminus (red). (b) Surface representation of the monomer structure of BpSDR. $(b)$ is in the same view as $(a)$ and shows access tunnels to the cofactor-binding cavity. (c) Topology of BpSDR. Helices are represented as cylinders, strands as arrows and loops as blue lines. The amino-terminus is labeled $\mathrm{N}$ and the carboxylterminus is labeled $\mathrm{C}$. 


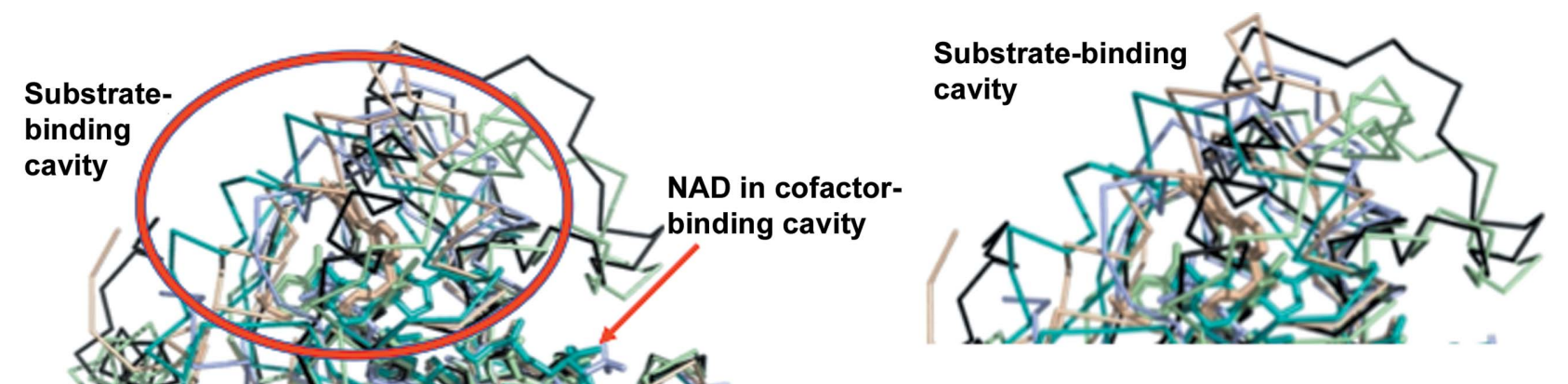

NAD in well conserved cofactor-binding cavity

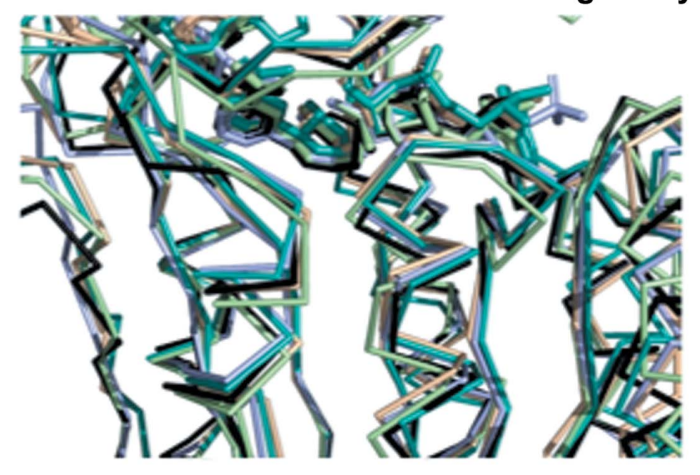

(a)
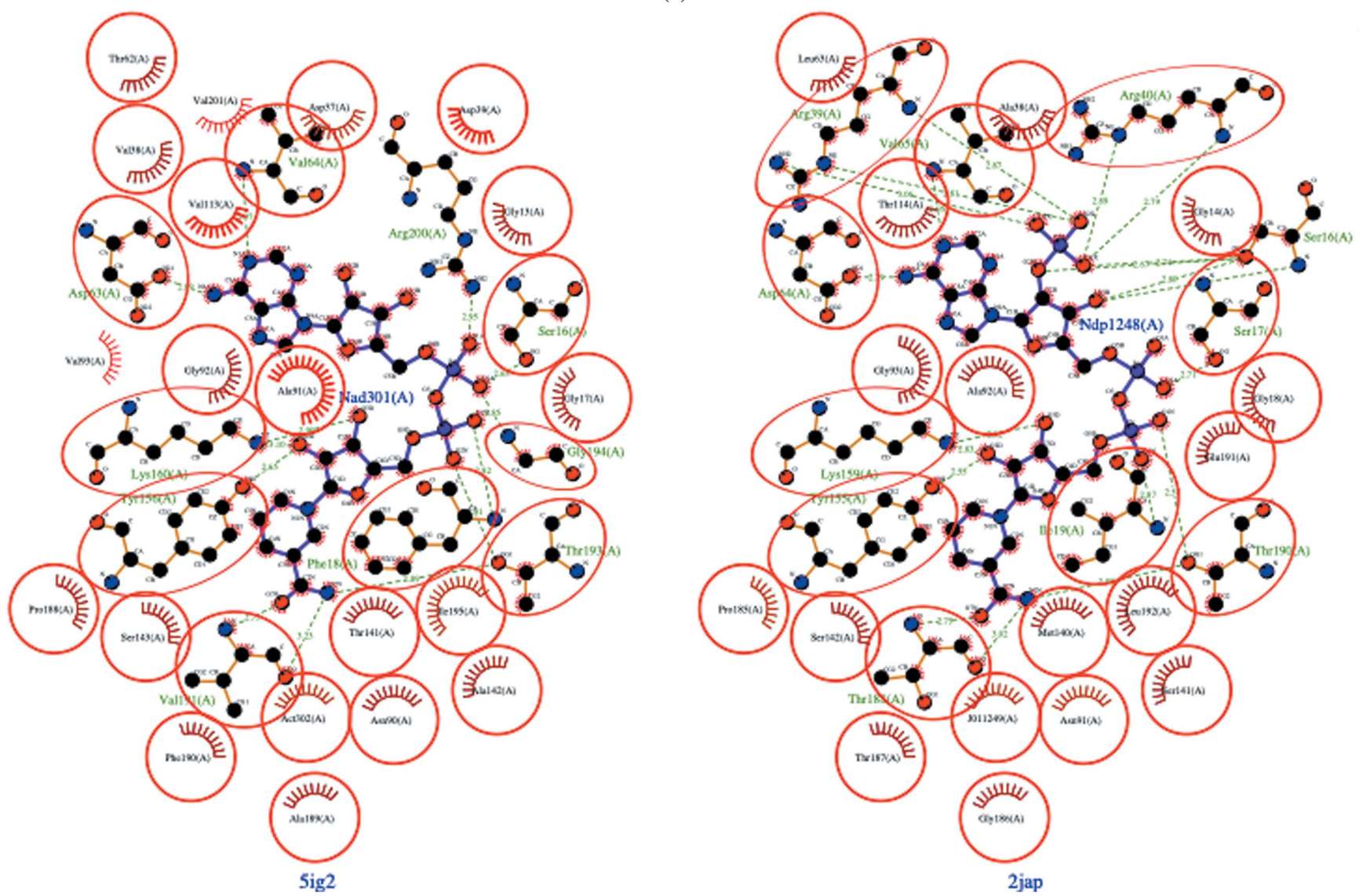

(b)

Figure 3

Comparison of representative SDRs with BpSDR. (a) SDRs have a well conserved NAD-binding region and a variable substrate-binding region which determines their specificity. The PDB codes for the monomers are $6 \mathrm{~g} 41$ (light orange), 2jap (light blue), 4yai (green), 1fmc (teal) and 5ig2 (black). (b) Side-by-side comparison of the NAD-binding domains of BpSDR (PDB entry 5ig2) with its closest structural neighbor the Rv0851c ortholog short-chain dehydrogenase from M. paratuberculosis (PDB entry 3tjr) reveal conserved residues involved in hydrogen bonds (dashed green lines); the hydrophobic interactions that predominate in the NAD-binding domain are shown as red arcs with lines. Corresponding atoms that are involved in hydrophobic contacts are shown in red circles. 
LIGPLOT analysis reveals a network of well conserved residues in the cofactor-binding domains (Fig. $3 b$ ). $B p$ SDR has less than $40 \%$ sequence identity to other known homologs (Fig. 4). However, it maintains the same overall topology as found in other SDRs. Its cofactor-binding domain is virtually identical to that in M. paratuberculosis (PDB entry 3tjr). Some conformational flexibility is observed in the substrate-binding domain and the carboxyl-terminus (Fig. 4). The variability in
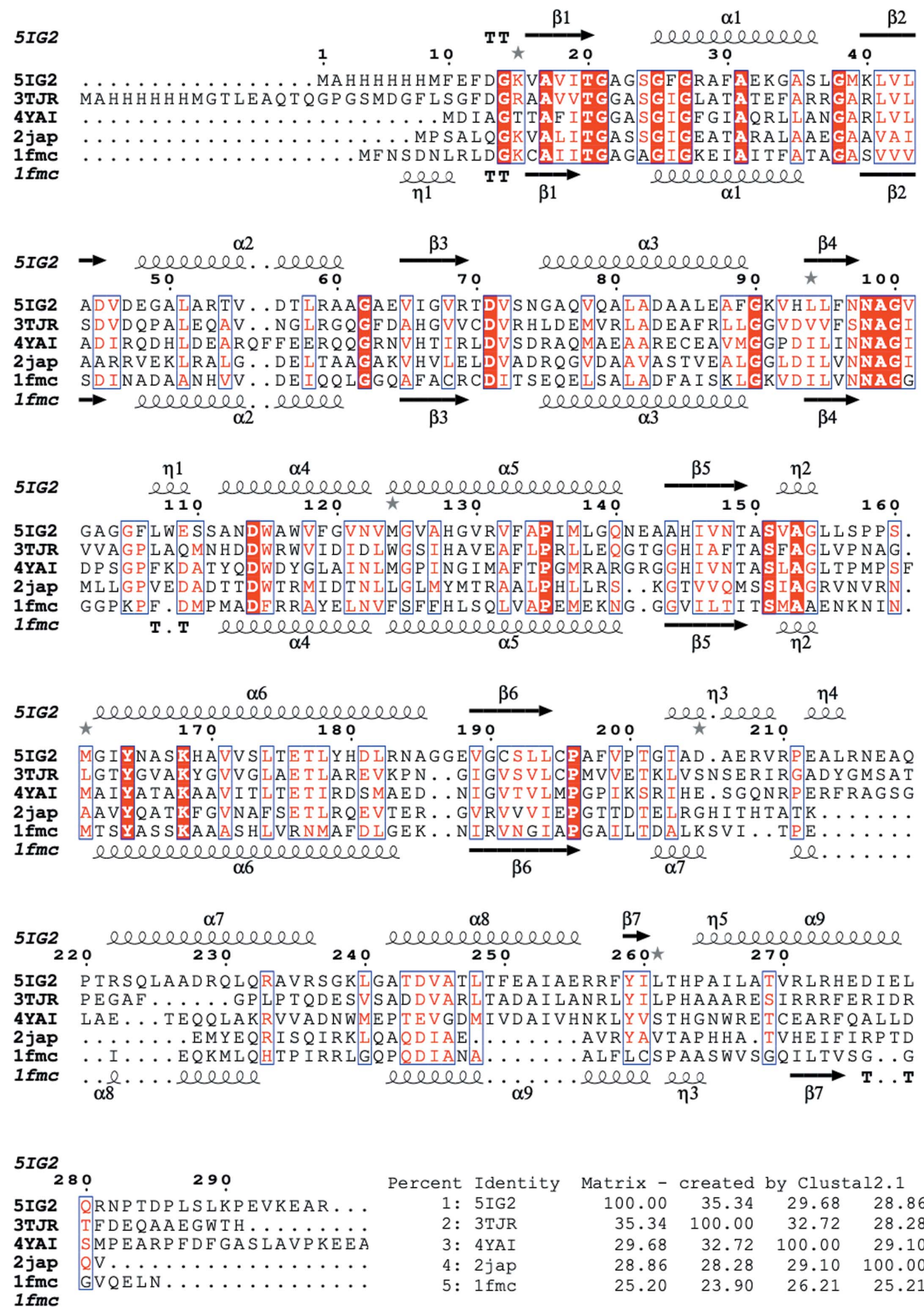

20290

QRNPTDPLSLKPEVKEAR . . .

TFDEQAAEGWTH ........

MPEARPFDFGASLAVPKEEA

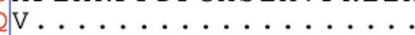

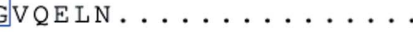

Percent Identity
1: 5 IG 2
$2: 3$ TJR
$3: 4$ YAI
$4: 2$ jap
$5: 1$ fmc

$\begin{array}{rrrr}\text { Matrix - created by Clustal2.1 } \\ 100.00 & 35.34 & 29.68 & 28.86 \\ 35.34 & 100.00 & 32.72 & 28.28 \\ 29.68 & 32.72 & 100.00 & 29.10 \\ 28.86 & 28.28 & 29.10 & 100.00 \\ 25.20 & 23.90 & 26.21 & 25.21\end{array}$

25.20

23.90

26.21

25.21

100.00

Figure 4

Structural and primary-sequence alignment of SDRs. The SDRs that are compared with BpSDR (PDB entry 5ig2) are the Rv0851c ortholog short-chain dehydrogenase from M. paratuberculosis (PDB entry 3tjr), the $7 \alpha$-hydroxysteroid dehydrogenase complex (PDB entry $1 \mathrm{fmc}$ ), Ligl from Sphingobium sp. strain SYK-6 (PDB entry 4yai), clavulanic acid dehydrogenase (PDB entry 2jap) and 17 $\beta$-hydroxysteroid dehydrogenase type 14 mutant Y253A (PDB entry 6g4l). The secondary-structure elements shown are $\alpha$-helices $(\alpha), 3_{10}$-helices $(\eta), \beta$-strands $(\beta)$ and $\beta$-turns (TT). Identical residues are shown in white on a red background and conserved residues are shown in red. This figure was generated using ESPript (Gouet et al., 1999, 2003). 
the substrate-binding domain may be exploited for the design of selective inhibitors or for the development of novel biocatalysts for chiral transformations.

\section{Conclusion}

$B p$ SDR has the prototypical SDR topology, a well conserved cofactor-binding site and structural differences in the carboxyl-terminus and substrate-binding regions. Our ongoing studies include studying structural differences between SDRs in order to identify selective inhibitors for SDRs from bacterial pathogens closely related to B. phymatum.

\section{Acknowledgements}

The SSGCID consortium is directed by PJM (principal investigator). It comprises many different scientists working at multiple centers towards determining the three-dimensional structures of proteins from biodefense organisms and emerging infectious diseases. In particular, we would like to thank the SSGCID cloning, protein-production and X-ray crystallography groups at the Center for Global Infectious Disease Research, the University of Washington and UCB. Hampton University's partnership with the SSGCID includes functional characterization and educational training. JA (MS, Hampton University) was sponsored by the Saudi Arabia Government and Northern Border University and supervised by OAA.

\section{Funding information}

This work was supported by federal funds from the National Institute of Allergy and Infectious Diseases (NIAID), National Institutes of Health (NIH), Department of Health and Human Services under Contract No. HHSN272201700059C from 1 September 2017 (SSGCID was funded under NIAID Contracts Nos. HHSN272201200025C from 1 September 2012 through 31 August 2017 and HHSN272200700057C from 28 September 2007 through 27 September 2012).

\section{References}

Asojo, O. A., Dranow, D. M., Serbzhinskiy, D., Subramanian, S., Staker, B., Edwards, T. E. \& Myler, P. J. (2018). Acta Cryst. F74, 294-299.

Asojo, O. A., Subramanian, S., Abendroth, J., Exley, I., Lorimer, D. D., Edwards, T. E. \& Myler, P. J. (2018). Acta Cryst. F74, 187-192.

Badran, M. J., Bertoletti, N., Keils, A., Heine, A., Klebe, G. \& Marchais-Oberwinkler, S. (2019). J. Steroid Biochem. Mol. Biol. 189, 135-144.

Baugh, L., Gallagher, L. A., Patrapuvich, R., Clifton, M. C., Gardberg, A. S., Edwards, T. E., Armour, B., Begley, D. W., Dieterich, S. H., Dranow, D. M., Abendroth, J., Fairman, J. W., Fox, D., Staker, B. L., Phan, I., Gillespie, A., Choi, R., Nakazawa-Hewitt, S., Nguyen, M. T., Napuli, A., Barrett, L., Buchko, G. W., Stacy, R., Myler, P. J., Stewart, L. J., Manoil, C. \& Van Voorhis, W. C. (2013). PLoS One, 8, e53851.

Baugh, L., Phan, I., Begley, D. W., Clifton, M. C., Armour, B., Dranow, D. M., Taylor, B. M., Muruthi, M. M., Abendroth, J., Fairman, J. W., Fox, D., Dieterich, S. H., Staker, B. L., Gardberg, A. S., Choi, R., Nakazawa Hewitt, S., Napuli, A. J., Myers, J., Barrett, L. K., Zhang, Y., Ferrell, M., Mundt, E., Thompkins, K., Tran, N., Lyons-Abbott, S., Abramov, A., Sekar, A., Serbzhinskiy, D., Lorimer, D., Buchko, G. W., Stacy, R., Stewart, L. J., Edwards, T. E., Van Voorhis, W. C. \& Myler, P. J. (2015). Tuberculosis, 95, 142-148.
Bryan, C. M., Bhandari, J., Napuli, A. J., Leibly, D. J., Choi, R., Kelley, A., Van Voorhis, W. C., Edwards, T. E. \& Stewart, L. J. (2011). Acta Cryst. F67, 1010-1014.

Chen, V. B., Arendall, W. B., Headd, J. J., Keedy, D. A., Immormino, R. M., Kapral, G. J., Murray, L. W., Richardson, J. S. \& Richardson, D. C. (2010). Acta Cryst. D66, 12-21.

Choi, R., Kelley, A., Leibly, D., Nakazawa Hewitt, S., Napuli, A. \& Van Voorhis, W. (2011). Acta Cryst. F67, 998-1005.

DeLano, W. L. (2002). PyMOL. http://www.pymol.org.

Emsley, P., Lohkamp, B., Scott, W. G. \& Cowtan, K. (2010). Acta Cryst. D66, 486-501.

Evans, P. (2006). Acta Cryst. D62, 72-82.

Gouet, P., Courcelle, E., Stuart, D. I. \& Métoz, F. (1999). Bioinformatics, 15, 305-308.

Gouet, P., Robert, X. \& Courcelle, E. (2003). Nucleic Acids Res. 31, 3320-3323.

Hall, C. M., Jaramillo, S., Jimenez, R., Stone, N. E., Centner, H., Busch, J. D., Bratsch, N., Roe, C. C., Gee, J. E., Hoffmaster, A. R., Rivera-Garcia, S., Soltero, F., Ryff, K., Perez-Padilla, J., Keim, P., Sahl, J. W. \& Wagner, D. M. (2019). PLoS Negl. Trop. Dis. 13, e0007727.

Kabsch, W. (2010). Acta Cryst. D66, 125-132.

Lebedev, A. A., Vagin, A. A. \& Murshudov, G. N. (2008). Acta Cryst. D64, 33-39.

Liebschner, D., Afonine, P. V., Baker, M. L., Bunkóczi, G., Chen, V. B., Croll, T. I., Hintze, B., Hung, L.-W., Jain, S., McCoy, A. J., Moriarty, N. W., Oeffner, R. D., Poon, B. K., Prisant, M. G., Read, R. J., Richardson, J. S., Richardson, D. C., Sammito, M. D., Sobolev, O. V., Stockwell, D. H., Terwilliger, T. C., Urzhumtsev, A. G., Videau, L. L., Williams, C. J. \& Adams, P. D. (2019). Acta Cryst. D75, 861-877.

MacKenzie, A. K., Kershaw, N. J., Hernandez, H., Robinson, C. V., Schofield, C. J. \& Andersson, I. (2007). Biochemistry, 46, 15231533.

Moulin, L., Klonowska, A., Caroline, B., Booth, K., Vriezen, J. A., Melkonian, R., James, E. K., Young, J. P., Bena, G., Hauser, L., Land, M., Kyrpides, N., Bruce, D., Chain, P., Copeland, A., Pitluck, S., Woyke, T., Lizotte-Waniewski, M., Bristow, J. \& Riley, M. (2014). Stand. Genomic Sci. 9, 763-774.

Myler, P. J., Stacy, R., Stewart, L., Staker, B. L., Van Voorhis, W. C., Varani, G. \& Buchko, G. W. (2009). Infect. Disord. Drug Targets, 9, 493-506.

Pereira, J. H., Heins, R. A., Gall, D. L., McAndrew, R. P., Deng, K., Holland, K. C., Donohue, T. J., Noguera, D. R., Simmons, B. A., Sale, K. L., Ralph, J. \& Adams, P. D. (2016). J. Biol. Chem. 291, 10228-10238.

Poe, R. H., Vassallo, C. L. \& Domm, B. M. (1971). Am. Rev. Respir. Dis. 104, 427-431.

Raymond, A., Haffner, T., Ng, N., Lorimer, D., Staker, B. \& Stewart, L. (2011). Acta Cryst. F67, 992-997.

Sawana, A., Adeolu, M. \& Gupta, R. S. (2014). Front. Genet. 5, 429. Serbzhinskiy, D. A., Clifton, M. C., Sankaran, B., Staker, B. L., Edwards, T. E. \& Myler, P. J. (2015). Acta Cryst. F71, 594-599.

Stacy, R., Begley, D. W., Phan, I., Staker, B. L., Van Voorhis, W. C., Varani, G., Buchko, G. W., Stewart, L. J. \& Myler, P. J. (2011). Acta Cryst. F67, 979-984.

Tanaka, N., Nonaka, T., Tanabe, T., Yoshimoto, T., Tsuru, D. \& Mitsui, Y. (1996). Biochemistry, 35, 7715-7730.

Vagin, A. \& Teplyakov, A. (2010). Acta Cryst. D66, 22-25.

Vandamme, P., Goris, J., Chen, W. M., de Vos, P. \& Willems, A. (2002). Syst. Appl. Microbiol. 25, 507-512.

Veluthat, C., Venkatnarayan, K., Padaki, P. \& Krishnaswamy, U. M. (2021). BMJ Case Rep 14, e242499.

Yabuuchi, E., Kosako, Y., Oyaizu, H., Yano, I., Hotta, H., Hashimoto, Y., Ezaki, T. \& Arakawa, M. (1992). Microbiol. Immunol. 36, 12511275.

Zhou-qi, C., Bo, Z., Guan-lin, X., Bin, L. \& Shi-wen, H. (2016). Rice Sci. 23, 111-118. 